\title{
Research Progress of Preoperative FPR, FAR or AFR in Patients with Colorectal Cancer
}

This article was published in the following Dove Press journal:

Cancer Management and Research

\section{Chen Chen \\ Yanlong Liu \\ Peng Han \\ Binbin Cui}

Department of Colorectal Surgery, Harbin Medical University Cancer

Hospital, Harbin, I5008I, Heilongjiang

Province, People's Republic of China
Correspondence: Binbin Cui

Department of Colorectal Surgery, Harbin Medical University Cancer

Hospital, No. 150 Raod Haping, Nangang

District, Harbin, I5008I, Heilongjiang

Province, People's Republic of China

Tel + I3351 I 12888

Email cbbhrb@I63.com
Abstract: Research has confirmed that plasma albumin (Alb), prealbumin (PA) and fibrinogen (Fib) are involved in regulating the occurrence and development of various tumors. Their levels in peripheral blood are related to the survival outcome and treatment response of patients, but the accuracy and specificity of single application have yet to be fully realized. A growing amount of evidence indicates that predictors such as preoperative fibrinogen to prealbumin ratio (FPR), fibrinogen to albumin ratio (FAR) or albumin to fibrinogen ratio (AFR) are emerging as comprehensive indicators. Indeed, their components play a key regulatory role in the progression of colorectal cancer (CRC). Preoperative FPR, FAR or AFR levels, therefore, are expected to become new biomarkers for prognosis evaluation and curative effect prediction for CRC patients and are significant in the guidance they could provide for the development of individualized treatment strategies.

Keywords: colorectal cancer, FPR, FAR or AFR, prognosis, efficacy

\section{Introduction}

Colorectal cancer (CRC) is the third most common malignant tumor in the world. Its incidence and mortality rates increase annually and are even rising among younger age groups. ${ }^{1,2}$ Based on the latest statistics, it is expected that in 2020, the number of new CRC cases in the United States will reach 147,950, and the number of deaths will exceed $53,200 .^{3}$ The continuous development of painless colonoscopy, targeted therapy and immunotherapy has greatly promoted the development of early screening, diagnosis and treatment of CRC, ${ }^{4,5}$ yet CRC, as a kind of molecular heterogeneous disease, is intractable Some studies have shown that the 5-year survival rate of CRC is about $32.8 \%-61.4 \%$. ${ }^{6,7}$ Such a range shows that there are differences in clinical treatment effects and that prognostic outcomes differ between patients. ${ }^{8}$ Therefore, more effective curative effect prediction and prognostic evaluation indicators will aid in stratifying the risk of CRC patients at an early stage, allowing for the formulation of accurate and individualized treatment plans as well as increased survival rates for patients.

At present, a variety of indicators are utilized to predict the prognosis of tumors, such as blood coagulation, inflammation, nutritional status, and immune status-related markers. ${ }^{9}$ Recently, more and more studies have suggested that the levels of plasma albumin (Alb), prealbumin (PA) and fibrinogen (Fib) are closely related to the prognosis of tumors. ${ }^{10-12}$ In addition, fibrinogen to prealbumin ratio (FPR) and fibrinogen to albumin ratio (FAR) or its reciprocal albumin to fibrinogen ratio (AFR) are emerging as comprehensive markers that can simultaneously reflect a patient's 
inflammation and nutritional status. These ratios also have shown medium and important value in the prognosis evaluation of many solid tumors, ${ }^{13}$ such as ovarian cancer, ${ }^{14}$ esophageal cancer, ${ }^{15}$ breast cancer, ${ }^{16}$ soft tissue sarcoma, ${ }^{17}$ gastric cancer ${ }^{18}$ and non-small cell lung cancer. ${ }^{19}$ For CRC patients, in particular, ${ }^{20,21}$ inflammation and nutrition are considered to be two important factors leading to tumor progression and in turn reduced survival rates. This article reviews the research progress of preoperative FPR and FAR or AFR levels in the areas of prognosis evaluation and efficacy prediction of CRC patients.

\section{The Structure and Function of Each Component of FPR and FAR or AFR}

FPR and FAR or AFR are the ratios of Fib to Alb and Fib to PA or PA to Fib, respectively. Therefore, the levels of $\mathrm{Alb}, \mathrm{PA}$ and Fib in peripheral blood are closely related to the levels of FPR and FAR or AFR. As is well known, Alb, PA, and Fib are currently recognized for the role they play as important indicators that affect the coagulation cascade and reflect one's nutritional status. They can also effectively reflect the local inflammation and systemic inflammation of a tumor. ${ }^{22-24}$ Their sources and functions are described below.

\section{The Source and Function of Albumin}

Albumin (Alb), which is mainly synthesized by hepatocytes, is the most abundant protein in human plasma, accounting for more than $50 \%{ }^{25}$ It not only participates in the maintenance of plasma colloidal osmotic pressure, the transportation of nutrients, the metabolism of toxic substances and other physiological processes but also can resist the invasion of microorganisms. This protein thus plays a key role in the body's acute and chronic inflammation. $^{26}$ The level of Alb acts as an important nutritional reference index for cancer patients. ${ }^{27}$ In addition, serum albumin levels in patients with locally advanced or metastatic malignancies will decrease regardless of malnutrition. ${ }^{28}$ Recent studies have proven that the preoperative peripheral blood Alb content has a nonnegligible value in the prognosis of CRC patients. ${ }^{29}$ The clinical study conducted by Gonzalez Trejo et al also confirmed this conclusion. ${ }^{30}$

\section{The Source and Function of Prealbumin}

The negative acute phase protein known as PA is synthesized by the liver and can sensitively reflect the degree of protein turnover in the body. With a short half-life of around two days, PA reflects the recent dietary intake of patients, rather than their overall nutritional status. ${ }^{31}$ It can reveal minor changes due to malnutrition in a considerably short period of time, as such, it can be regarded as the main nutritional detection index of high-risk patients. ${ }^{32} \mathrm{PA}$, also known as transthyretin, is involved in regulating the synthesis and transportation of vitamin A and thyroxine. ${ }^{33}$ Its serum concentration may be affected by various factors, such as nutritional intake, inflammatory state, liver disease, endocrine disease, etc. ${ }^{34,35}$ In addition, PA also has thymic properties, which can enhance the body's immunity by promoting the maturation of lymphocytes and exerting potential anti-tumor effects. ${ }^{11}$ However, current studies have shown that low preoperative PA levels are an independent poor prognostic factor for cancer-specific survival. ${ }^{36}$

\section{The Source and Function of Fibrinogen}

Fib, also known as coagulation factor 1 , is the highest coagulation factor in the blood. It is synthesized by the liver and has a half-life of four to six days. Damage to vascular endothelial cells can result in the activation of the endogenous and exogenous coagulation system, the promotion of platelet aggregation, and an increase in blood viscosity. Under the action of thrombin, Fib is transformed into fibrin, which plays a central role in thrombosis. ${ }^{37,38}$ As an acute phase protein, Fib is not only abnormally expressed in coagulation-related diseases but also increases in varying degrees when the body undergoes operations, infections, trauma, and tumors. ${ }^{39}$ Previous studies have shown that Fib as a key inflammatory regulator, it is connected to the processes of malignant tumor cell proliferation, angiogenesis, invasion and metastasis, inflammatory microenvironment formation and the level of inflammation. ${ }^{40}$ Recent meta-analysis results also show that Fib levels are significantly correlated with the prognosis of a variety of gastrointestinal tumors. ${ }^{41}$

\section{The Functions of FPR and FAR or AFR Components in CRC}

Over the years, many scholars have studied and analyzed the levels of Fib, Alb, and PA in the peripheral blood of patients with malignant tumors as well as healthy people. Their findings indicate that the levels of Fib in cancer patients are generally higher than normal, while the levels of Alb and PA are relatively low. ${ }^{40,42}$ Hyperfibrinogenemia and hypoalbuminemia are frequently observed in CRC 
patients, especially individuals with metastases..$^{23,43}$ The following subsections detail the functions of preoperative FPR and FAR or AFR components in coagulation function, nutritional metabolism, inflammation, tumor cell proliferation and adhesion, and tumor angiogenesis in CRC patients.

\section{Affect Coagulation Function}

The occurrence, development, and treatment of a variety of malignant tumors are often accompanied by one or more coagulation disorders. The activation of the coagulation system and the release of coagulation-related factors will promote thrombosis, and the hypercoagulable state of the blood leads to malignant tumor proliferation, invasion, and metastasis. ${ }^{44}$ Studies have shown that approximately $50 \%$ of tumor patients who have not metastasized and $90 \%$ of metastatic tumor patients have a certain degree of coagulation abnormality. ${ }^{45,46}$ Furthermore, the hyperactivity of the coagulation and fibrinolysis system may be the key material basis for inducing tumor progression.

Studies have also found that the presence of malignant tumors in patients significantly increases the level of Fib, which increases blood viscosity and peripheral resistance and causes the body to enter a long-term hypercoagulable state. ${ }^{47}$ The interaction between high level Fib and cytokines secreted by tumor cells can play a synergistic effect, leading to further promotion of red blood cell adhesion and thrombosis. ${ }^{48}$ Therefore, Fib can be considered as a key determinant of tumor metastasis potential. ${ }^{18}$ The study of Hong et al confirmed that the high level of Fib in peripheral blood pre-surgery predicts a poor prognosis for CRC patients. ${ }^{49}$ In addition, the activation of Fib changes the values of FPR and FAR or AFR and results in abnormal hemorheology, the acceleration of thrombosis, ${ }^{50}$ ultimately increases the likelihood of perioperative pulmonary embolism occurring. The risk of cardiovascular and cerebrovascular embolism and other diseases may cause patients to miss the best window of opportunity for surgery or prolong the recovery period after surgery.

\section{Affect Nutrition Metabolism}

Patients with malignant tumors often experience symptoms of malnutrition, such as progressive weight loss. Such systems create a vicious cycle wherein the defense capabilities of the immune system are weakened, the risk of infection increases, and the proliferation and development of tumor cells accelerate. The cycle then causes a continuous deterioration of the cancer patient's condition and is accompanied with a decline in one's quality of life. ${ }^{51} \mathrm{~A}$ number of studies have shown that nutritional levels are closely related to tumor prognosis. ${ }^{52,53}$ The occurrence and progression of CRC closely correlate with an impaired immune defense and surveillance function due to a patient's poor nutritional status, ${ }^{54-56}$ especially in those with metastases. ${ }^{57}$ In addition, some studies have shown that nutritional status is related to the efficacy and toxicity of patients receiving neoadjuvant therapy. ${ }^{58,59}$

Alb level is a sensitive nutritional index. ${ }^{60}$ Excessive proliferation of tumor cells is one of the key characteristics of malignant tumors. This usually requires an increase in the uptake of albumin in order to cells to maintain their metabolic levels, which will lead to a decrease in Alb storage capacity and in turn hypoalbuminemia. When compared to patients with other cancers, those with gastrointestinal tumors often have gastrointestinal obstruction or malabsorption, ${ }^{61}$ as such the risk of hypoalbuminemia is higher. ${ }^{62}$ The changes in vascular permeability caused by tumors can also transfer Alb inside blood vessels to the extravascular stroma and induce pleural and peritoneal effusions. ${ }^{63}$ Previous studies have shown that hypoalbuminemia is closely related to the occurrence of various postoperative complications, ${ }^{64}$ such as anastomotic edema, anastomotic leakage and an extension of the time required for tissue repair. These complications will result in poor prognosis. ${ }^{11,65}$ This is consistent with the results of Lee et al. ${ }^{34}$ In the analysis of factors affecting the prognosis of CRC patients, Sun et al also found that low PA is an independent risk factor affecting postoperative survival. ${ }^{19}$ Because tumor cell proliferation and migration depend on the host to provide nutritional support, some scholars noted that malnutrition in patients with metastatic CRC can lead to prolonged hospital stays, poor prognosis and poor quality of life, which is an important determinant factor. ${ }^{66}$

\section{Affect Inflammation}

An inflammatory response also plays an important role in tumor progression. The microenvironment of a tumor includes various inflammatory-related cells, namely stromal cells, cytokines, and tumor cells. ${ }^{67}$ These important components not only promote the occurrence and development of tumors but also inhibit the immune system's ability to kill the tumor, reduce the therapeutic response of a tumor to cytotoxic drugs, and ultimately impact a patient's survival rate. ${ }^{68-70}$ Studies have shown that 
inflammation is an obvious feature of $\mathrm{CRC}$, which exists at all stages of the occurrence and development of CRC. ${ }^{71}$ Inflammation can trigger the mutation of proto-oncogene and tumor suppressor genes in CRC patients, induce the secondary organs and tissues to enter the pre-metastasis state, and provide tumor cells with a microenvironment, where they can easily metastasize. Continuous use of lowdose NSAIDs can reduce the risk of CRC. ${ }^{72,73}$ More and more evidence indicates that systemic inflammatory response markers correlate with the long-term survival of a variety of tumors ${ }^{74,75}$ and serve as prognostic predictor. $^{76-79}$

At present, research has demonstrated that serum PA levels will change rapidly when inflammation and tissue damage occur and are related to the nature and scope of inflammation and damage. ${ }^{80}$ The tumor can induce the release of a variety of pro-inflammatory factors, inhibit the synthesis of $\mathrm{Alb}$, and reduce the amount of Alb. Thus, a change in Alb levels is directly proportional to the patient's level of inflammation. ${ }^{81}$ Wei Shenghong and others determined that Alb is a key factor involved in systemic inflammatory response. Down-regulation of Alb expression can enhance tumor-related inflammatory response and release a series of cytokines that contribute to tumor progression. ${ }^{82}$ Therefore, the levels of Alb and PA in the peripheral blood not only can represent the nutritional status of the body but also reflect the degree of inflammation in the body. In addition, Fib increases in systemic inflammation. The C-terminal of Fib $\gamma$ chain can interact with CD11b or CD11c/CD18 integrin receptors of inflammatory cells to activate and increase the recruitment and infiltration of inflammatory cells, such as monocytes, macrophages, neutrophils, $\mathrm{T}$ cells and $\mathrm{B}$ cells in tumor microenvironments. The recruitment and infiltration of inflammatory cells up-regulate the secretion of interleukin-6 (IL-6), IL-21 and IL-33. That secretion in turn activates $\mathrm{NF}-\kappa \mathrm{B}$ and JAK/STAT pathways, enhances the intensity of systemic inflammatory response, and affects the recovery and survival of patients. ${ }^{83,84}$

\section{Affect the Proliferation, Adhesion, and Angiogenesis of Tumor Cells}

As is commonly understood, the growth and migration of malignant tumors are based on cell proliferation, survival, adhesion, and the formation of new blood vessels. The occurrence of tumor invasion and metastasis often indicates poor prognosis. An increasing number of $\mathrm{CRC}$ - related studies also show that Fib, Alb and PA are closely linked to patient prognosis, ${ }^{20,85,86}$ and CRC patients are more susceptible to progression and metastasis where the expression of Fib was upregulated, while the expression of Alb and PA was downregulated. ${ }^{87}$

It has been found that Fib acts as a bridge that connects platelets and circulating tumor cells to binding proteins and is also an important mediator of tumor cell proliferation. Fib can bind with endothelial cell adhesion molecule1 and various growth factors (such as FGF, VEGF and TGF-b) to maintain or enhance the adhesion function and survival status of micrometastasis in target organs. The blood vessels of target organs have the ability to release proliferation signals, ${ }^{19}$ thus regulating the proliferation, adhesion, survival and apoptosis of tumor cells. ${ }^{88}$ Additionally, fib is a degradation product of fibrin, and fibrin acts as an important component in the formation of the extracellular matrix skeleton. Therefore, an increase of Fib indicates the destruction of the extracellular matrix structure and the occurrence of invasion or metastasis. ${ }^{89}$ When a Fib deficiency is present, the proliferation and dissemination of CRC and other solid tumors are substantially slowed down. ${ }^{90}$ Fib can also regulate the expression of E-cadherin and vimentin and induce epithelialmesenchymal transition (EMT) through the Akt/mTOR pathway, thereby enhancing the adhesion and migration abilities of tumor cells. ${ }^{91}$ Further, a study by Zhao et al found that tumor cells can produce additional endogenous Fib, which leads to an increase in the Fib content. Moreover, the combination of Fib and fibroblasts can promote the formation of neovascularization and provide nutritional support along with a favorable microenvironment for tumor recurrence or metastasis. ${ }^{92}$

In order to maintain the ability for unlimited proliferation and growth, malignant tumors require a continuous supply of nutritional energy. Alb and PA, as common nutritional evaluation indicators, are closely related to the occurrence and progress of tumors. Moreover, some studies have noted that Alb has the function of maintaining DNA replication and cell stable proliferation. It can inhibit the expression of tumor necrosis factor alpha (TNF- $\alpha$ ), upregulate the expression of vascular cell adhesion molecule-1, and reduce the apoptosis of endothelial cells, thus contributing to the proliferation, adhesion and metastasis of tumor cells, all of which ultimately leads to poor prognosis. $^{37}$

The uncontrolled proliferation and metastasis of malignant tumors are also affected by $\mathrm{T}$ cell-mediated tumor 
immune killing and tumor immune escape within a microenvironment. PA can induce lymphocyte maturation in the thymus, ${ }^{11}$ resulting in the maturation of $\mathrm{T}$ cells that will migrate to the blood and peripheral lymphoid tissue. At this point, they will further differentiate into CD4 + T cells or CD8 + T cells. These cells then perform specific cellular immunity and immunoregulation functions, form a suppressed or activated immune microenvironment, and regulate the proliferation and metastasis of tumor cells. ${ }^{93}$

\section{Application of FPR and FAR or AFR in CRC}

During the course of the disease, measuring Alb, PA, and Fib serum levels will indicate the presence of cancer cells in the majority of cancer patients, particularly those with CRC. Examining a patient's liver function and coagulation function is routine tests for both preoperative examinations and postoperative reviews. Measuring the serum levels of FPR, FAR or AFR has the following advantages: (1) The test method is easy to conduct, will not bring discomfort or pain to patients, reduces the psychological burden of patients, and greatly increases compliance; (2) The cost of detection is low and does not increase the economic burden of patients; (3) The test is safe and repeatable without side effects; (4) It is easy to popularize, which is conducive to achieving hierarchical diagnosis and treatment, rational allocation of medical resources, and reduces unnecessary trips for patients to receive medical treatment; (5) The detection cycle is short, and the results can be obtained quickly. Generally, the results may be obtained in $1-2$, which allow for the timely preliminary judgement of the patient's condition and provide guidance in providing a diagnosis and adjusting the treatment plan.

Currently, although much research has shown that Alb, $\mathrm{PA}$ and Fib are related to the prognosis and treatment effect of cancer patients, some scholars question the accuracy and effectiveness of using them alone. ${ }^{94,95}$ FPR and FAR are emerging comprehensive biomarkers. Low AFR and high FPR or FAR are associated with an increased risk of mortality and a risk of recurrence for multiple malignancies. ${ }^{17,94,96}$ Reports have shown that preoperative FPR and FAR or AFR can predict the prognosis of malignant tumors, such as CRC, non-small cell lung cancer, esophageal cancer, breast cancer, hepatocellular carcinoma, gallbladder cancer and gastric cancer. Their expression levels correlate with the therapeutic effects of radiotherapy and chemotherapy. ${ }^{15,16,18,65,97-105}$ In recent years, an increasing number of scholars have highlighted the application value of FPR and FAR or AFR in colorectal cancer treatment. The detection of serum levels of FPR and FAR or AFR is not only suitable for CRC patients with AJCC diagnosed as being stage I-III but also for high-risk patients with metastasis. These tests assist in evaluating the prognosis of patients and predicting the curative effects of adjuvant radiotherapy and chemotherapy, which in turn serves to guide the selection of the proper treatment scheme and realize the individualized treatment goal. The related studies are shown in Table 1.

\section{Application of FPR and FAR or AFR in Prognosis Assessment}

Sun et al ${ }^{106}$ recently measured the neutrophil to lymphocyte ratio (NLR), FPR and FAR in 455 newly diagnosed CRC patients, 455 benign colorectal polyps patients, and 455 healthy people. Although the ratios of NLR, FAR, and FPR in CRC patients were significantly higher than those in healthy people, further ROC curve analysis revealed that the diagnostic effect of FAR and FPR surpassed that of NLR. However, when FPR, CEA and CA199 were combined to diagnose $\mathrm{CRC}$, the maximum area under the ROC curve (AUC) was 0.845 , specificity was $85.3 \%$, sensitivity was $67.9 \%$, positive predictive value was $83.5 \%$ and the negative predictive value was $70.9 \%$. It was concluded that FPR could be used as an effective biomarker for CRC diagnosis. Sun et $\mathrm{al}^{19}$ subsequently conducted an in-depth study on 702 patients with resectable CRC to evaluate the prognostic value of AFR, FPR, Fib, Alb, PA, and a novel carcinoembryonic antigen (CEA)-carbohydrate antigen 19-9 (CA199)-FPR (CCF) score. The results showed that high FPR was significantly associated with the survival rate of CRC patients, and the CCF score was better than FPR, CEA, CA199, CEACA199 (CCI), and CEA-FPR (CFI) scores in predicting prognosis. Based on this research, it can be concluded that FPR and CCF are able to serve as effective biomarkers for predicting prognosis of colorectal cancer. Chen et $\mathrm{al}^{20}$ conducted a stratified cohort study on 990 patients who were first diagnosed with metastatic CRC, during which time the levels of circulating inflammatory cells, Fib, Alb, PA, AFR, and FPR were measured. It was found that FPR is an independent prognostic factor of left-side metastatic CRC; an increase in its value indicates the decrease of progression-free survival and overall survival. However, 


\begin{tabular}{|c|c|c|c|c|c|c|c|c|}
\hline  & 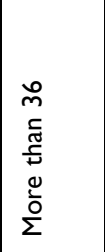 & 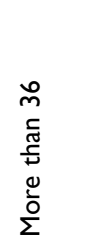 & 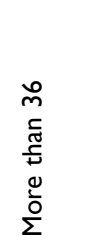 & 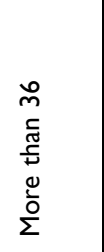 & 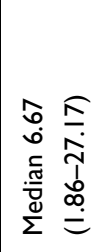 & $\mid \begin{array}{c}\infty \\
\tilde{T} \\
\tilde{\underline{w}} \\
\underline{\tilde{w}} \\
\Sigma\end{array}$ & 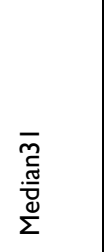 & 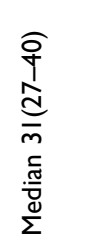 \\
\hline 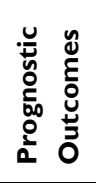 & os & 。ั & $\begin{array}{l}\text { un } \\
\text { ws } \\
\text { ot }\end{array}$ & $\begin{array}{l}\frac{m}{a} \\
\text { के }\end{array}$ & 뜸 & 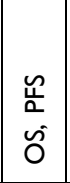 & o & 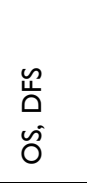 \\
\hline 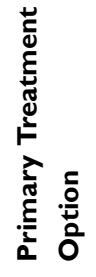 &  & 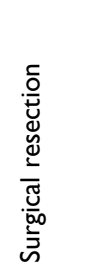 & 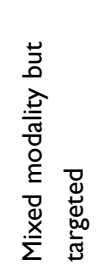 & 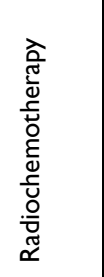 & 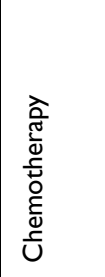 & 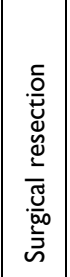 &  & 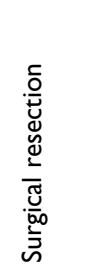 \\
\hline 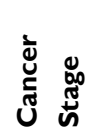 & 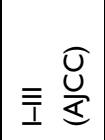 & $=\frac{\bar{U}}{\underline{\underline{U}}}$ & 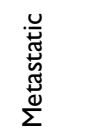 & 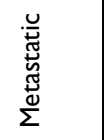 &  & 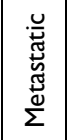 & $\overline{\bar{I}} \frac{\widehat{U}}{\underline{s}}$ & $\geq \frac{U}{I}$ \\
\hline 怤 & ฉี & 虽 & 多 & R & $\pi$ & 字 & $\stackrel{\infty}{\sim}$ & $\stackrel{\infty}{\infty}$ \\
\hline 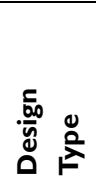 & 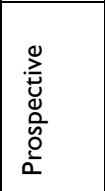 & 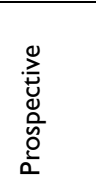 & 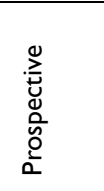 & 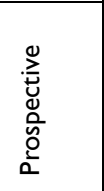 & 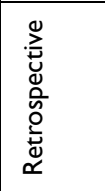 & 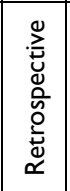 & 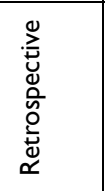 & 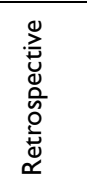 \\
\hline 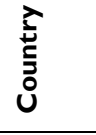 & 苞 & $\begin{array}{l}\frac{\tilde{c}}{\tilde{z}} \\
\end{array}$ & 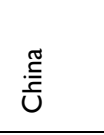 & $\begin{array}{l}\frac{\pi}{\tilde{z}} \\
\end{array}$ & 趇 & 莺 & 急 & 氶 \\
\hline 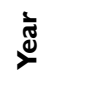 & $\frac{\infty}{a}$ & $\frac{\infty}{i}$ & के & iे & $\frac{o}{\bar{i}}$ & $\frac{a}{\bar{i}}$ & సి & ڤ̊ \\
\hline  & $\begin{array}{l}\tilde{N} \\
\alpha \\
\alpha \\
\infty \\
\infty \\
\underline{\infty}\end{array}$ & $\begin{array}{l}\tilde{N} \\
\alpha \\
\infty \\
\infty \\
\underline{\infty}\end{array}$ & 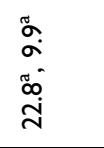 & 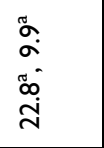 & 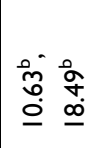 & \begin{tabular}{|l|} 
\\
0 \\
0 \\
0 \\
0
\end{tabular} & 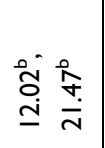 & 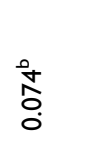 \\
\hline 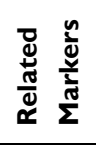 & 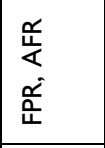 &  & 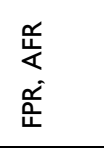 & 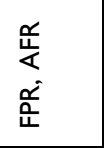 & $\begin{array}{l}\frac{\alpha}{0.0} \\
\frac{0}{4} \\
\frac{\alpha}{4}\end{array}$ & $\underset{\mathbb{L}}{\mathbb{a}}$ & $\begin{array}{l}\frac{d}{\mathrm{O}} \\
\mathrm{u} \\
\frac{\tilde{r}}{<}\end{array}$ & $\frac{\alpha}{4}$ \\
\hline 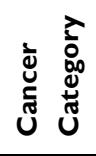 & $\ddot{U}$ & Ư & $\mathscr{U}$ & Ư & Ư & $\begin{array}{l}\sum_{\mathcal{U}} \\
\text { Un }\end{array}$ & Ư & $\ddot{U}$ \\
\hline 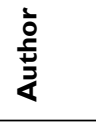 & $\stackrel{\frac{a}{\bar{c}}}{\bar{\sigma}}$ & $\stackrel{\frac{a}{\bar{\sigma}}}{\bar{\sigma}}$ &  & 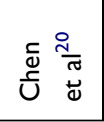 &  & $\mid \begin{array}{l}\bar{s}_{00} \\
\bar{c}_{0} \\
\bar{s}\end{array}$ & 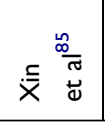 & 兽 \\
\hline
\end{tabular}


for patients with right-side metastatic CRC, FPR has no association with survival. Recently, a study by Yachen et $\mathrm{al}^{87}$ included 286 patients with CRC who underwent radical resection. The study statistically analyzed the effects of preoperative AFR and FPR values on the prognostic survival of patients. Although according to the area under the ROC curve, AFR and FPR both offer good predictive value for prognosis $(\mathrm{P}<0.05)$, the results of multivariate analysis show that only preoperative FPR has good predictive ability for the prognosis of CRC patients. Furthermore, preoperative FPR has the potential to become one of the necessary indicators for prognosis evaluation. FAR was determined to be related to the prognosis of CRC after an analysis of the clinical data of 386 patients with colorectal cancer radical resection was conducted by Shao Shengli and others. The team highlighted that FAR has a good predictive value for the prognosis of patients with radical resection. ${ }^{107}$ In addition, Wang et $\mathrm{al}^{21}$ analyzed the prognosis value of NLR, lymphocyte-monocyte ratio (LMR), platelet-lymphocyte ratio (PLR), systemic immune-inflammation index (SII, derived from lymphocyte, neutrophil, and platelet counts), and CEA in 452 patients with colorectal cancer liver metastasis (CRLM) who were undergoing hepatectomy. The best cutoff point of FAR was $7.6 \%$. The level of FAR is superior to other inflammatory markers and CEA in predicting the overall survival (OS) and disease-free survival (DFS) of patients, indicating that preoperative FAR is an independent prognostic indicator of colorectal cancer patients with liver metastasis after hepatectomy.

\section{Application of FPR and FAR or AFR in Efficacy Prediction}

So far, a plethora of studies have reported the relationship between the chemotherapy response in CRC patients and pretreatment albumin-globulin ratio, neutrophillymphocyte ratio, lymphocyte-monocyte ratio as well as platelet-lymphocyte ratio. ${ }^{108-111}$ As predictive markers for the efficacy of radiotherapy and chemotherapy, FPR and FAR or AFR have been applied to patients with gastric cancer and non-small cell lung cancer and have shown promising results. ${ }^{18,98}$ These applications show that FPR and FAR or AFR have great potential in the selection of optimal treatment options and prediction of efficacy. Sun et $\mathrm{al}^{19}$ conducted a 3-year follow-up of 702 patients with stage I-III CRC undergoing radical resection. The results showed that patients with stage II-III CRC with high FPR or CCF (scored $\geq 1$ ) were more likely to benefit from adjuvant chemotherapy than patients with low FPR or CCF (scored $=0$ ). FPR and CCF scores are promising classification biomarkers that indicate a patient with stage II-III CRC will benefit from adjuvant chemotherapy. The study of Chen et $\mathrm{al}^{20}$ revealed the relationship between FPR and prognosis of CRC patients. Furthermore, their research noted that although CRC patients with high FPR usually had chemoradiotherapy resistance, these patients could benefit from chemoradiotherapy combined with bevacizumab. For patients with low FPR, palliative resection combined with chemoradiotherapy could result in longer survival periods. In 2019, Zhang et $\mathrm{al}^{32}$ published a retrospective study that collected the clinical data of 71 patients with metastatic CRC. For this study, the albuminto-globulin ratio (AGR), FAR, prealbumin-to-globulin ratio (PGR), FPR, NLR, LMR, and PLR were monitored. The results showed that FPR was slightly larger than FAR and AGR was larger than PGR in AUC. However, these mixed results did not demonstrate that PGR and FPR were better than AGR and FAR in predicting prognosis. The AUCs of NLR, LMR, and PLR were smaller and not statistically significant. This being the case, only AGR, FAR, PGR and FPR were eventually selected as prognostic factors as they possessed important predictive value for metastatic CRC patients receiving first-line chemotherapy.

The above studies suggest that FPR and FAR or AFR have great clinical application prospects. They can not only be applied to the prognosis assessment of CRC patients but also can be used as effective markers to stratify the benefit groups of adjuvant chemoradiotherapy and predict the therapeutic effect, so as to achieve the ultimate goal of individualized precise treatment. However, further research is needed on the application of FPR and FAR or AFR alone or in combination with other indicators as well as which markers can be utilized to a greater extent.

\section{Conclusion}

In recent years, the incidence and mortality of CRC in China have ranked third and fifth in malignant tumors that severely threaten people's health, respectively. ${ }^{112}$ Finding efficient and specific $\mathrm{CRC}$ curative effect prediction and prognostic evaluation indicators will aid in clinical monitoring and early intervention of postoperative recurrence or metastasis, improve patient curative effect and quality of life, and enhance prognosis. Although Fib, Alb and PA can predict the prognosis and treatment effect of tumor patients to 
a certain extent, there are limitations in the accuracy and specificity of a single index. Therefore, the joint application of multiple indicators plays a crucial, more efficient prediction role and has become a general trend. As emerging comprehensive biomarkers, FPR and FAR or AFR offer significant advantages due to their simplicity, availability, economic advantages, and reliability. They can accurately distinguish CRC patients who are suitable for adjuvant chemotherapy and radiotherapy and provide the best treatment plan. Their ability to effectively predict the progress of CRC patients and help to screen out patients with high risk of poor prognosis, making them invaluable for the development of effective treatment plans. Preoperative FPR and FAR or AFR are expected to become irreplaceable detection markers in CRC curative effect prediction and prognosis evaluation and guide the development of clinical individualized treatment. Although the application value of preoperative FPR and FAR or AFR in the field of CRC is increasing, there are still great differences between the experimental results of different centers. Therefore, it is necessary to conduct a wider range of multi-center clinical trials and conduct more detailed stratified studies of samples to further confirm their predictive role in CRC.

\section{Ethical Approval and Ethical Standards}

The study was performed in accordance with the Declaration of Helsinki. This study does not contain any studies with human participants or animals performed by any of the authors.

\section{Informed Consent}

Informed consent is not required for this study.

\section{Funding}

General program of National Natural Science Foundation of China (82072640); Youth program of National Natural Science Foundation of China (81702766); Natural Science Foundation of Heilongjiang Province (ZD2017019).

\section{Disclosure}

The authors declare no potential conflicts of interest.

\section{References}

1. Barontini J, Antinucci M, Tofanelli S, et al. Association between polymorphisms of TAS2R16 and susceptibility to colorectal cancer. BMC Gastroenterol. 2017;17(1):104.
2. Stoffel EM, Koeppe E, Everett J, et al. Germline genetic features of young individuals with colorectal cancer. Gastroenterology. 2018;154(4):897-905.e1.

3. Siegel RL, Miller KD, Goding Sauer A, et al. Colorectal cancer statistics, 2020. CA Cancer J Clin. 2020;70(3):145-164.

4. Bergheim J, Semaan A, Gevensleben H, et al. Potential of quantitative SEPT9 and SHOX2 methylation in plasmatic circulating cell-free DNA as auxiliary staging parameter in colorectal cancer: a prospective observational cohort study. Br J Cancer. 2018;118 (9):1217-1228.

5. Singal AG, Gupta S, Skinner CS, et al. Effect of colonoscopy outreach vs fecal immunochemical test outreach on colorectal cancer screening completion: a randomized clinical trial. JAMA. 2017;318(9):806-815.

6. Kennedy A, Cohn M, Coldwell DM, et al. Updated survival outcomes and analysis of long-term survivors from the MORE study on safety and efficacy of radioembolization in patients with unresectable colorectal cancer liver metastases. $J$ Gastrointest Oncol. 2017;8(4):614-624.

7. Park S, Kang BW, Lee SJ, et al. Clinical significance of systemic chemotherapy after curative resection of metachronous pulmonary metastases from colorectal cancer. Cancer Chemother Pharmacol. 2017;80(1):187-193.

8. Inamura K. Colorectal cancers: an update on their molecular pathology. Cancers (Basel). 2018;10(1).

9. Sun KY, Xu JB, Chen SL, et al. Novel immunological and nutritional-based prognostic index for gastric cancer. World J Gastroenterol. 2015;21(19):5961-5971.

10. Cai W, Kong W, Dong B, et al. Pretreatment serum prealbumin as an independent prognostic indicator in patients with metastatic renal cell carcinoma using tyrosine kinase inhibitors as first-line target therapy. Clin Genitourin Cancer. 2017;15(3): e437-e446.

11. Miura K, Hamanaka K, Koizumi T, et al. Clinical significance of preoperative serum albumin level for prognosis in surgically resected patients with non-small cell lung cancer: comparative study of normal lung, emphysema, and pulmonary fibrosis. Lung Cancer. 2017;111:88-95.

12. Huang W, Wang S, Zhang H, et al. Prognostic significance of combined fibrinogen concentration and neutrophil-to-lymphocyte ratio in patients with resectable non-small cell lung cancer. Cancer Biol Med. 2018;15(1):88-96.

13. Li SQ, You XH, Sun F, et al. Albumin to fibrinogen ratio and fibrinogen to pre-albumin ratio are economical, simple and promising prognostic factors for solid malignancy. $J$ Thorac Dis. 2019;11(Suppl 15):S2036-2036S2038.

14. Zhang WW, Zhang ZW, He L. Analysis of prognostic factors in 342 patients with ovarian cancer undergoing surgery. Chin J Cancer Prev Treat. 2019;26:1288-1295. doi:10.16073/j.cnki. cjept.2019.17.11

15. Tan Z, Zhang M, Han Q, et al. A novel blood tool of cancer prognosis in esophageal squamous cell carcinoma: the fibrinogen/ albumin ratio. $J$ Cancer. 2017;8(6):1025-1029.

16. Hwang KT, Chung JK, Roh EY, et al. Prognostic influence of preoperative fibrinogen to albumin ratio for breast cancer. $J$ Breast Cancer. 2017;20(3):254-263.

17. Liang Y, Wang W, Que Y, et al. Prognostic value of the fibrinogen/albumin ratio (FAR) in patients with operable soft tissue sarcoma. BMC Cancer. 2018;18(1):942.

18. Zhang J, Li SQ, Liao ZH, et al. Prognostic value of a novel FPR biomarker in patients with surgical stage II and III gastric cancer. Oncotarget. 2017;8(43):75195-75205.

19. Sun F, Peng HX, Gao QF, et al. Preoperative circulating FPR and CCF score are promising biomarkers for predicting clinical outcome of stage II-III colorectal cancer patients. Cancer Manag Res. 2018;10:2151-2161. 
20. Chen QG, Zhang L, Sun F, et al. Elevated FPR confers to radiochemoresistance and predicts clinical efficacy and outcome of metastatic colorectal cancer patients. Aging (Albany NY). 2019;11(6):1716-1732.

21. Wang YY, Liu ZZ, Xu D, et al. Fibrinogen-Albumin Ratio Index (FARI): a more promising inflammation-based prognostic marker for patients undergoing hepatectomy for colorectal liver metastases. Ann Surg Oncol. 2019;26(11):3682-3692.

22. Allin KH, Bojesen SE, Nordestgaard BG. Inflammatory biomarkers and risk of cancer in 84,000 individuals from the general population. Int J Cancer. 2016;139(7):1493-1500.

23. Nazha B, Moussaly E, Zaarour M, et al. Hypoalbuminemia in colorectal cancer prognosis: nutritional marker or inflammatory surrogate? World J Gastrointest Surg. 2015;7(12):370-377.

24. Zhou J, Hiki N, Mine S, et al. Role of prealbumin as a powerful and simple index for predicting postoperative complications after gastric cancer surgery. Ann Surg Oncol. 2017;24(2):510-517.

25. Loftus TJ, Brown MP, Slish JH, Rosenthal MD. Serum levels of prealbumin and albumin for preoperative risk stratification. Nutr Clin Pract. 2019;34(3):340-348.

26. Li XF, Wang X, Lu WJ, et al. Relationship between fibrinogen/ albumin ratio and coronary collateral circulation in patients with chronic total occlusion. Henan Med Res. 2020;29:589.

27. Miao Y, Li S, Yan Q, et al. Prognostic significance of preoperative prognostic nutritional index in epithelial ovarian cancer patients treated with platinum-based chemotherapy. Oncol Res Treat. 2016;39(11):712-719.

28. Deme D, Telekes A. [Prognostic importance of albumin in oncology]. Orv Hetil. 2018;159(3):96-106. Hungarian.

29. Fujino S, Myoshi N, Saso K, et al. The inflammation-nutrition score supports the prognostic prediction of the TNM stage for colorectal cancer patients after curative resection. Surg Today. 2020;50(2):163-170.

30. González-Trejo S, Carrillo JF, Carmona-Herrera DD, et al. Baseline serum albumin and other common clinical markers are prognostic factors in colorectal carcinoma: a retrospective cohort study. Medicine (Baltimore). 2017;96(15):e6610.

31. Lu J, Xu BB, Zheng ZF, et al. CRP/prealbumin, a novel inflammatory index for predicting recurrence after radical resection in gastric cancer patients: post hoc analysis of a randomized Phase III trial. Gastric Cancer. 2019;22(3):536-545.

32. Zhang L, Zhang J, Wang Y, et al. Potential prognostic factors for predicting the chemotherapeutic outcomes and prognosis of patients with metastatic colorectal cancer. J Clin Lab Anal. 2019;33(8):e22958.

33. Smith SH. Using albumin and prealbumin to assess nutritional status. Nursing. 2017;47(4):65-66.

34. Lee JL, Oh ES, Lee RW, et al. Serum albumin and prealbumin in calorically restricted, nondiseased individuals: a systematic review. Am J Med. 2015;128(9):1023.e1-22.

35. Liu X, Sun X, Liu J, et al. Preoperative C-reactive protein/albumin ratio predicts prognosis of patients after curative resection for gastric cancer. Transl Oncol. 2015;8(4):339-345.

36. Ma YQ, Zhang YQ, Liu DL, et al. Correlation analysis of postoperative albumin changes and complications in elderly patients with gastric cancer with normal preoperative serum albumin level. Clin Oncol China. 2020;47:823.

37. Sun YR, Gao XY, Lu ZJ, et al. Predictive efficacy of preoperative plasma fibrinogen to albumin ratio for adverse cardiovascular events in patients with NSTE-ACS after PCI. Shandong Med. 2020;60:81.

38. Kavitha S, Sridhar MG, Satheesh S. Periprocedural plasma fibrinogen levels and coronary stent outcome. Indian Heart J. 2015;67 (5):440-443.

39. Ahn HJ, Baker SK, Norris EH, et al. Inflaming the Brain. Neuron. 2019;101(6):991-993.
40. Perisanidis C, Psyrri A, Cohen EE, et al. Prognostic role of pretreatment plasma fibrinogen in patients with solid tumors: a systematic review and meta-analysis. Cancer Treat Rev. 2015;41(10):960-970.

41. Ji R, Ren Q, Bai S, et al. Prognostic significance of pretreatment plasma fibrinogen level in patients with digestive system tumors: a meta-analysis. Int J Biol Markers. 2018;33(3):254-265.

42. Huang J, Wang Y, Yuan Y, et al. Preoperative serum pre-albumin as an independent prognostic indicator in patients with localized upper tract urothelial carcinoma after radical nephroureterectomy. Oncotarget. 2017;8(22):36772-36779.

43. $\mathrm{Hu} \mathrm{WH}$, Cajas-Monson LC, Eisenstein S, et al. Preoperative malnutrition assessments as predictors of postoperative mortality and morbidity in colorectal cancer: an analysis of ACS-NSQIP. Nutr J. 2015;14:91.

44. Marco P, Marco A. [Biomolecular markers in cancer-associated thromboembolism]. Med Clin (Barc). 2015;144(Suppl 1):21-25. Spain.

45. Zhang D, Zhou X, Bao W, et al. Plasma fibrinogen levels are correlated with postoperative distant metastasis and prognosis in esophageal squamous cell carcinoma. Oncotarget. 2015;6 (35):38410-38420.

46. Jain A, Zhang Q, Toh HC. Awakening immunity against cancer: a 2017 primer for clinicians. Chin J Cancer. 2017;36(1):67.

47. Zhu L, Zhang JQ. Clinical analysis of coagulation and fibrinolysis indexes in patients with malignant tumor and relevant nursing countermeasures. Thrombosis Hemostasis. 2016;22:588.

48. Li RT, Sun ZQ, Wang HB. The prognostic value of the ratio of fibrinogen to prealbumin in patients with advanced non-small cell lung cancer. J Clin Oncol. 2019;24:237.

49. Hong T, Shen D, Chen X, et al. Preoperative plasma fibrinogen, but not D-dimer might represent a prognostic factor in non-metastatic colorectal cancer: a prospective cohort study. Cancer Biomark. 2017;19(1):103-111.

50. Zhou F, Wen GR. Relationship between fibrinogen/albumin ratio, high sensitivity $\mathrm{C}$-reactive protein/prealbumin ratio and prognosis of patients with acute coronary syndrome after percutaneous coronary intervention. Trauma Crit Care Med. 2020;8:113.

51. Virizuela JA, Camblor-álvarez M, Luengo-Pérez LM, et al. Nutritional support and parenteral nutrition in cancer patients: an expert consensus report. Clin Transl Oncol. 2018;20(5):619-629.

52. Okada S, Shimada J, Kato D, et al. Clinical significance of prognostic nutritional index after surgical treatment in lung cancer. Ann Thorac Surg. 2017;104(1):296-302.

53. Torre LA, Bray F, Siegel RL, et al. Global cancer statistics, 2012. CA Cancer J Clin. 2015;65(2):87-108.

54. You SY, Rui W, Chen ST, et al. Process of immunogenic cell death caused by disulfiram as the anti-colorectal cancer candidate. Biochem Biophys Res Commun. 2019;513(4):891-897.

55. Hänggi K, Oncogenic RB. KRAS drives immune suppression in colorectal cancer. Cancer Cell. 2019;35(4):535-537.

56. Kotoula V, Fostira F, Fountzilas E. Primary resistance to immune checkpoint inhibitors in metastatic colorectal cancer-beyond the misdiagnosis. JAMA Oncol. 2019;5(5):740-741.

57. Leischner C, Burkard M, Pfeiffer MM, et al. Nutritional immunology: function of natural killer cells and their modulation by resveratrol for cancer prevention and treatment. Nutr J. 2016;15(1):47.

58. Matsumoto Y, Zhou Q, Kamimura K, et al. The prognostic nutrition index predicts the development of hematological toxicities in and the prognosis of esophageal cancer patients treated with cisplatin plus 5-fluorouracil chemotherapy. Nutr Cancer. 2018;70(3):447-452.

59. Nakatani M, Migita K, Matsumoto S, et al. Prognostic significance of the prognostic nutritional index in patients with recurrent esophageal squamous cell carcinoma. Nutr Cancer. 2018;70 (3):467-473. 
60. Daniele A, Divella R, Abbate I, et al. Assessment of nutritional and inflammatory status to determine the prevalence of malnutrition in patients undergoing surgery for colorectal carcinoma. Anticancer Res. 2017;37(3):1281-1287.

61. Magzal F, Sela S, Szuchman-Sapir A, et al. In-vivo oxidized albumin- a pro-inflammatory agent in hypoalbuminemia. PLoS One. 2017;12(5):e0177799.

62. Alkan A, Köksoy EB, Utkan G. Albumin to globulin ratio, a predictor or a misleader? Ann Oncol. 2015;26(2):443-444.

63. Moujaess E, Fakhoury M, Assi T, et al. The Therapeutic use of human albumin in cancer patients' management. Crit Rev Oncol Hematol. 2017;120:203-209.

64. Filip B, Scarpa M, Cavallin F, et al. Postoperative outcome after oesophagectomy for cancer: nutritional status is the missing ring in the current prognostic scores. Eur J Surg Oncol. 2015;41 (6):787-794.

65. Xu Q, Yan Y, Gu S, et al. A novel inflammation-based prognostic score: the fibrinogen/albumin ratio predicts prognoses of patients after curative resection for hepatocellular carcinoma. J Immunol Res. 2018;2018:4925498.

66. Barao K, Abe Vicente Cavagnari M, Silva Fucuta P, et al. Association between nutrition status and survival in elderly patients with colorectal cancer. Nutr Clin Pract. 2017;32 (5):658-663.

67. Mills JC, Samuelson LC. Past questions and current understanding about gastric cancer. Gastroenterology. 2018;155(4):939-944.

68. Che Y, Wang J, Li Y, et al. Cisplatin-activated PAI-1 secretion in the cancer-associated fibroblasts with paracrine effects promoting esophageal squamous cell carcinoma progression and causing chemoresistance. Cell Death Dis. 2018;9(7):759.

69. Bakker E, Qattan M, Mutti L, et al. The role of microenvironment and immunity in drug response in leukemia. Biochim Biophys Acta. 2016;1863(3):414-426.

70. Isella C, Terrasi A, Bellomo SE, et al. Stromal contribution to the colorectal cancer transcriptome. Nat Genet. 2015;47(4):312-319.

71. Farhan-Alanie OM, McMahon J, McMillan DC. Systemic inflammatory response and survival in patients undergoing curative resection of oral squamous cell carcinoma. Br J Oral Maxillofac Surg. 2015;53(2):126-131.

72. Friis S, Riis AH, Erichsen R, et al. Low-dose aspirin or nonsteroidal anti-inflammatory drug use and colorectal cancer risk: a population-based, case-control study. Ann Intern Med. 2015;163 (5):347-355

73. Nan H, Hutter CM, Lin Y, et al. Association of aspirin and NSAID use with risk of colorectal cancer according to genetic variants. JAMA. 2015;313(11):1133-1142.

74. Dolan RD, McSorley ST, Park JH, et al. The prognostic value of systemic inflammation in patients undergoing surgery for colon cancer: comparison of composite ratios and cumulative scores. $\mathrm{Br}$ $J$ Cancer. 2018;119(1):40-51.

75. Jagadesham VP, Lagarde SM, Immanuel A, et al. Systemic inflammatory markers and outcome in patients with locally advanced adenocarcinoma of the oesophagus and gastro-oesophageal junction. Br J Surg. 2017;104(4):401-407.

76. Berzigotti A, Reig M, Abraldes JG, et al. Portal hypertension and the outcome of surgery for hepatocellular carcinoma in compensated cirrhosis: a systematic review and meta-analysis. Hepatology. 2015;61(2):526-536.

77. Kozasa K, Mabuchi S, Komura N, et al. Comparison of clinical utilities of the platelet count and platelet-lymphocyte ratio for predicting survival in patients with cervical cancer: a single institutional study and literature review. Oncotarget. 2017;8(33):55394-55404.

78. Wu Y, Zhou D, Zhang G, et al. Preoperative serum platelet-lymphocyte ratio as a prognostic factor in cholangiocarcinoma patients after radical resection: a retrospective analysis of 119 patients. Gastroenterol Res Pract. 2019;2019:8506967.
79. Wu CC, $\mathrm{Li} \mathrm{SH}, \mathrm{Lu} \mathrm{HI}$, et al. Inflammation-based prognostic scores predict the prognosis of locally advanced cervical esophageal squamous cell carcinoma patients receiving curative concurrent chemoradiotherapy: a propensity score-matched analysis. PeerJ. 2018;6:e5655.

80. Lv Y, Zhang J, Liu Z, et al. A novel inflammation-based prognostic index for patients with esophageal squamous cell carcinoma: neutrophil lymphocyte ratio/prealbumin ratio. Medicine (Baltimore). 2019;98(7):e14562.

81. Wei XL, Wang FH, Zhang DS, et al. A novel inflammation-based prognostic score in esophageal squamous cell carcinoma: the C-reactive protein/albumin ratio. BMC Cancer. 2015;15:350.

82. Wei SH, Wang Y, Ye ZS, et al. Relationship between preoperative serum albumin level and clinicopathological features and prognosis of patients with gastric stump cancer. Chin J Gen Surg. 2018;33:828.

83. Huynh PT, Beswick EJ, Coronado YA, et al. CD90(+) stromal cells are the major source of IL-6, which supports cancer stem-like cells and inflammation in colorectal cancer. Int J Cancer. 2016;138(8):1971-1981.

84. Li Y, Shi J, Qi S, et al. Correction to: IL-33 facilitates proliferation of colorectal cancer dependent on COX2/PGE2. J Exp Clin Cancer Res. 2020;39(1):15.

85. Lee S, Huh SJ, Oh SY, et al. Clinical significance of coagulation factors in operable colorectal cancer. Oncol Lett. 2017;13 (6):4669-4674.

86. Lee JH, Hyun JH, Kim DY, et al. The role of fibrinogen as a predictor in preoperative chemoradiation for rectal cancer. Ann Surg Oncol. 2015;22(1):209-215.

87. Yachen X, Qiqi W, Xia W. Prognostic evaluation of preoperative AFR and FPR in patients with colorectal cancer. J Clin Pathol Res. 2020;40(4):898-904.

88. Zhang Y, Xiao G. Prognostic significance of the ratio of fibrinogen and albumin in human malignancies: a meta-analysis. Cancer Manag Res. 2019;11:3381-3393.

89. Schreuders EH, Ruco A, Rabeneck L, et al. Colorectal cancer screening: a global overview of existing programmes. Gut. 2015;64(10):1637-1649.

90. Adams GN, Rosenfeldt L, Frederick M, et al. Colon cancer growth and dissemination relies upon thrombin, stromal PAR-1, and fibrinogen. Cancer Res. 2015;75(19):4235-4243.

91. Li R, Song SB, Li Q, et al. Effect of the ratio of fibrinogen to albumin before operation on the prognosis of patients with gallbladder carcinoma. Chin J Gen Surg. 2020;35:142.

92. Zhao $\mathrm{C}, \mathrm{Su} \mathrm{Y}$, Zhang J, et al. Fibrinogen-derived fibrinostatin inhibits tumor growth through anti-angiogenesis. Cancer Sci. 2015;106(11):1596-1606.

93. Chen C, Liu Y, Cui B. Effect of radiotherapy on T cell and PD-1/ PD-L1 blocking therapy in tumor microenvironment. Hum Vaccin Immunother. 2021;1-13.

94. Xu WY, Zhang HH, Xiong JP, et al. Prognostic significance of the fibrinogen-to-albumin ratio in gallbladder cancer patients. World J Gastroenterol. 2018;24(29):3281-3292.

95. Graf ME, Sookthai D, Johnson T, et al. Pre-diagnostic plasma concentrations of Fibrinogen, sGPIIb/IIIa, sP-selectin, sThrombomodulin, Thrombopoietin in relation to cancer risk: findings from a large prospective study. Int J Cancer. 2018;143 (11):2659-2667.

96. Tominaga T, Nonaka T, Sumida Y, et al. The C-reactive protein to albumin ratio as a predictor of severe side effects of adjuvant chemotherapy in stage III colorectal cancer patients. PLoS One. 2016;11(12):e0167967.

97. Lu S, Liu Z, Zhou X, et al. Preoperative Fibrinogen-Albumin Ratio Index (FARI) is a reliable prognosis and chemoradiotherapy sensitivity predictor in locally advanced rectal cancer patients undergoing radical surgery following neoadjuvant chemoradiotherapy. Cancer Manag Res. 2020;12:8555-8568. 
98. Li SQ, Jiang YH, Lin J, et al. Albumin-to-fibrinogen ratio as a promising biomarker to predict clinical outcome of non-small cell lung cancer individuals. Cancer Med. 2018;7(4):1221-1231.

99. Gao QF, Qiu JC, Huang XH, et al. The predictive and prognostic role of a novel ADS score in esophageal squamous cell carcinoma patients undergoing esophagectomy. Cancer Cell Int. 2018;18:153.

100. Zhang L, Chen QG, Li SQ, et al. Preoperative fibrinogen to prealbumin ratio as a novel predictor for clinical outcome of hepatocellular carcinoma. Future Oncol. 2019;15(1):13-22.

101. Wu M, Pan Y, Jia Z, et al. Preoperative plasma fibrinogen and serum albumin score is an independent prognostic factor for resectable stage II-III gastric cancer. Dis Markers. 2019;2019:9060845.

102. Patel M, McSorley ST, Park JH, et al. The relationship between right-sided tumour location, tumour microenvironment, systemic inflammation, adjuvant therapy and survival in patients undergoing surgery for colon and rectal cancer. $\mathrm{Br} J$ Cancer. 2018;118(5):705-712.

103. Lee GH, Malietzis G, Askari A, et al. Is right-sided colon cancer different to left-sided colorectal cancer? - a systematic review. Eur J Surg Oncol. 2015;41(3):300-308.

104. Matsuda S, Takeuchi H, Kawakubo H, et al. Cumulative prognostic scores based on plasma fibrinogen and serum albumin levels in esophageal cancer patients treated with transthoracic esophagectomy: comparison with the Glasgow prognostic score. Ann Surg Oncol. 2015;22(1):302-310.

105. Sun DW, An L, Lv GY. Albumin-fibrinogen ratio and fibrinogen-prealbumin ratio as promising prognostic markers for cancers: an updated meta-analysis. World J Surg Oncol. 2020;18 (1):9.
106. Sun F, Tan YA, Gao QF, et al. Circulating fibrinogen to pre-albumin ratio is a promising biomarker for diagnosis of colorectal cancer. J Clin Lab Anal. 2019;33(1):e22635.

107. Shao SL, Chen YM, Yan HP, Feng YD. A novel prognostic marker for colorectal cancer: preoperative fibrinogen albumin ratio. J Huazhong Univ Sci Technol. 2020;49(3):249-254.

108. Shibutani M, Maeda K, Nagahara H, et al. The pretreatment albumin to globulin ratio predicts chemotherapeutic outcomes in patients with unresectable metastatic colorectal cancer. $B M C$ Cancer. 2015;15:347.

109. Artaç M, Uysal M, Karaağaç M, et al. Prognostic impact of neutrophil/lymphocyte ratio, platelet count, CRP, and albumin levels in metastatic colorectal cancer patients treated with FOLFIRI-bevacizumab. $J$ Gastrointest Cancer. 2017;48 (2):176-180

110. Shibutani M, Maeda K, Nagahara H, et al. Prognostic significance of the lymphocyte-to-monocyte ratio in patients with metastatic colorectal cancer. World J Gastroenterol. 2015;21 (34):9966-9973.

111. Wu Y, Li C, Zhao J, et al. Neutrophil-to-lymphocyte and platelet-to-lymphocyte ratios predict chemotherapy outcomes and prognosis in patients with colorectal cancer and synchronous liver metastasis. World J Surg Oncol. 2016;14(1):289.

112. Zheng RS, Sun KX, Zhang SW, et al. [Report of cancer epidemiology in China, 2015]. Zhonghua Zhong Liu Za Zhi. 2019;41 (1):19-28. Chinese.

\section{Publish your work in this journal}

Cancer Management and Research is an international, peer-reviewed open access journal focusing on cancer research and the optimal use of preventative and integrated treatment interventions to achieve improved outcomes, enhanced survival and quality of life for the cancer patient.
The manuscript management system is completely online and includes a very quick and fair peer-review system, which is all easy to use. Visit http://www.dovepress.com/testimonials.php to read real quotes from published authors. 